Received: 29 August 2018

Accepted: 23 April 2019

Published online: 10 May 2019
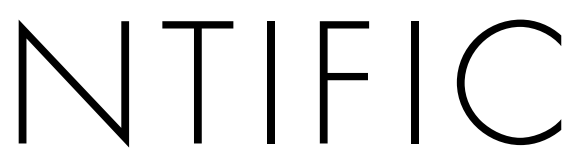
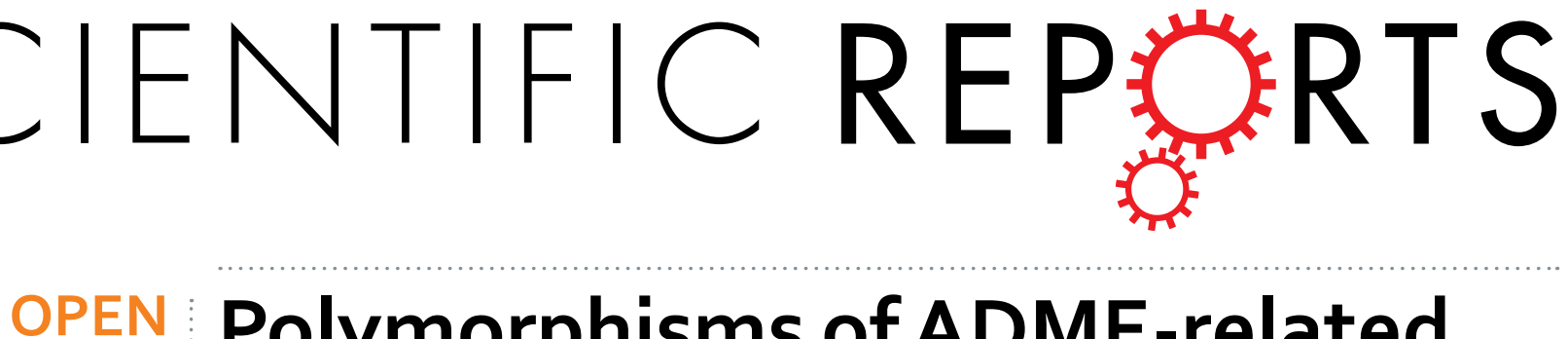

\title{
Polymorphisms of ADME-related genes and their implications for drug safety and efficacy in Amazonian Amerindians
}

Juliana Carla Gomes Rodrigues ${ }^{1}$, Marianne Rodrigues Fernandes ${ }^{1}$, João Farias Guerreiro ${ }^{2}$, Artur Luiz da Costa da Silva $\mathbb{1}^{3}$, Ândrea Ribeiro-dos-Santos $\mathbb{1}^{1,2}$, Sidney Santos ${ }^{1,2}$ \& Ney Pereira Carneiro dos Santos ${ }^{1,2}$

The variation in the allelic frequencies of polymorphic pharmacogenes among different ethnic groups may be responsible for severe adverse reactions to or altered efficacy of a wide variety of drugs. Amazonian Amerindian populations have a unique genetic profile that may have a fundamental on the efficacy and safety of certain drugs. The genetic characteristics of these populations are poorly known, which can negatively impact the systematic application of treatments guided by pharmacogenomic guidelines. We investigated the diversity of 32 polymorphisms in genes responsible for drug Absorption, Distribution, Metabolism and Excretion (ADME) in Amazonian Amerindians, and compared the findings with populations from other continents available in the 1000 Genomes database. We found significantly different $(P \leq 1.56 \mathrm{E}-03)$ allelic frequencies and genotype distributions in many study markers in comparison with African, European, American and Asian populations. Based on FST values, the Amerindian population was also the most distinct (mean FST $=0.09917$ ). These data highlight the unique genetic profile of the indigenous population from the Brazilian Amazon region, which is potentially important from a pharmacogenetic viewpoint. Understanding the diversity of ADMErelated genetic markers is crucial to the implementation of individualized pharmacogenomic treatment protocols in Amerindian populations, as well as populations with a high degree of admixture with this ethnic group, such as the general Brazilian population.

Inter-individual variability plays a fundamental role in the tolerance of and response to a number of different treatments that are widely used in clinical practice ${ }^{1}$. Much of this inter-individual variability is related to polymorphisms in the genes involved in the Absorption, Distribution, Metabolism, and Excretion (the ADME process) of drugs or the mechanisms that determine their action in the body ${ }^{2,3}$.

The discovery that the response to and tolerance of drugs may vary not only among individuals, but systematically among populations, in particular among different ethnic groups, had a fundamental impact on pharmacogenetic studies, leading to the development of the field of Pharmacoethnicity ${ }^{4-6}$. The principal determinant of this interethnic variability is the allelic frequencies of the polymorphisms found in pharmacogenes, which may vary considerably among different ethnic groups ${ }^{7-9}$.

The Brazilian population is characterized by its high degree of ethnic admixture ${ }^{10}$, being formed by three principal ancestral groups: European settlers, African slaves, and Amerindians (Native Americans). Five centuries of miscegenation of these groups have resulted in an extremely heterogeneous genetic makeup, and a considerable challenge for pharmacogenetic studies in Brazil ${ }^{11-13}$.

According to the most recent census (2010) of the Brazilian Institute of Geography and Statistics ${ }^{14}$ the Amerindian population of Brazil numbers 896 917, representing $0.47 \%$ of the country's population. While this

${ }^{1}$ Research Center of Oncology, Federal University of Pará, Belém, Brazil. ${ }^{2}$ Laboratory of Human and Medical Genetics, Institute of Biological Science, Federal University of Pará, Belém, Brazil. ${ }^{3}$ Genomics and Bioinformatics Laboratory, Institute of Biological Science, Federal University of Pará, Belém, Brazil. Juliana Carla Gomes Rodrigues and Marianne Rodrigues Fernandes contributed equally. Correspondence and requests for materials should be addressed to N.P.C.d.S. (email: npcsantos@yahoo.com.br) 
population is genetically distinct, few data are available on the incorporation of modern habits (industrialized foodstuffs, synthetic drugs, alcoholic beverages, etc.) into its traditional lifestyles. It nevertheless seems likely that distinct patterns of toxicity and response to pharmacological therapies will be found in this ethnic group in comparison with other populations ${ }^{15,16}$. However, genomic studies of pharmacogenetic biomarkers are extremely rare in Amazonian Amerindian populations.

The general lack of pharmacogenomic data for these Amerindian populations represents a major obstacle to the incorporation of Pharmacogenomics (PGx) into the development of individualized treatment protocols designed to maximize the efficacy of therapies in this ethnic group. In this context, the identification of genetic polymorphisms and molecular markers of clinical significance in these populations will be essential for the development of effective treatment protocols not only for this ethnic group, but also for the populations that are admixed with this group, such as the Brazilian one ${ }^{17}$.

In this context, the present study investigated a set of 32 molecular ADME markers in a combined Amazonian Amerindian population, and compared the results with populations representing five continents, obtained from the 1000 Genomes database.

\section{Material and Methods}

Study Populations. The study population was composed of 146 healthy individuals from three Amerindian groups located in the Amazon region of Brazil, selected from a sample database of an epidemiological study of indigenous populations of Pará State. This group included 61 individuals from the Asurini do Trocará group, 25 from the Asurini do Koatinemo group, and 60 from the Kayapó-Xicrin group. The study was approved by the National Committee for Ethics in Research (CONEP), with CAAE number 20654313.6.0000.5172. The informed consent was obtained from each study participant and all research methods in this study were performed in accordance with the approved guidelines.

For the analyses, these three populations were combined in a single group, denominated IND. For comparison with populations from other continents, we used data obtained from the 1000 Genomes, phase 3 release (available at http://www.1000genomes.org), composed of 661 individuals from Africa (AFR), 503 (SAS).

from Europe (EUR), 347 from the Americas (AMR), 504 from East Asia (EAS), and 489 from South Asia

Selection of Markers. Thirty-two polymorphisms of 16 pharmacogenes were selected based on three main criteria: (1) the marker must be involved in any of the steps of the ADME process; (2) the PGx biomarker should also have high-level clinical annotations or related VIPs in the public database of the PharmGKB (www. pharmgkb.org), or it has been pointed in the specific literature as an important biomarker (www.ncbi.nlm.nih. gov/pubmed), and (3) it should also be among the PGx biomarkers recommended for drug dosage adjustment by global drug regulatory agencies.

After all the three criteria were applied, we have selected 11 membrane transporter gene polymorphisms, 11 polymorphisms of phase I metabolizing genes, and 10 polymorphisms of other genes that are indirectly involved in the ADME process.

Genotyping and Quality Control. The DNA was extracted from the peripheral blood of the 146 study subjects using the commercial Biopur Mini Spin Plus-250 kit (Biopur, Brazil), according to the manufacture's recommendations. The concentration and purity of the DNA were measured with a NanoDrop 1000 spectrophotometer (Termo Fisher Scientific, Wilmington, DE).

The polymorphisms of the ADME-related genes were genotyped by allelic discrimination using the TaqMan OpenArray Genotyping technology, with a set of 32 customized assays, which were run in a QuantStudio ${ }^{\mathrm{TM}} 12 \mathrm{~K}$ Flex Real-Time PCR system (Applied Biosystems, Life Technologies, Carlsbad, USA), according to the manufacturer's protocol. The Taqman Genotyper software was used for data analysis and to verify the accuracy of the genotype readings, as well as the quality of the genotyping. The data were then filtered for SNPs that deviated from Hardy-Weinberg equilibrium (HWE).

Statistical analysis. The allele frequencies of the Amerindian population were obtained directly by gene counting, and compared with the other study populations (AFR, EUR, AMR, EAS, and SAS). The distribution of the genotypes among the six populations was analyzed using Fisher's exact test. Pairwise comparisons between populations were also based on Fisher's exact test, with the p-value being adjusted by the Bonferroni correction, with a critical p-value of $1.56 \mathrm{E}-03$ being considered in all cases.

The inter-population variability of the ADME SNPs was assessed using Wright's fixation index $\left(\mathrm{F}_{\mathrm{ST}}\right)$. We ran a multidimensional scaling analysis of the $\mathrm{F}_{\mathrm{ST}}$ values to provide a graphical representation of the genetic differentiation of the Amerindian population in comparison with each of the five study populations. The analyses were run in SPSS v.12.0 (SPSS Inc., Chicago, IL) and Arlequin v.3.5 ${ }^{18}$.

\section{Results}

The three Amazonian Amerindian populations were analyzed together (IND) for the comparisons of allele and genotype frequencies, and the $\mathrm{F}_{\mathrm{ST}}$ values with the five populations from the 1000 Genomes Project database (AFR, AMR, EAS, EUR, and SAS). The allele frequencies were determined for a total of 32 markers (Table 1). The Hardy-Weinberg Equilibrium (HWE) was calculated and p-values of less than 1.56E-03 were considered significant. Six of the 32 markers investigated in the Amerindian population were not in HWE - rs9524885 of the ABCC4 gene, rs8192726 of the CYP2A6 gene, rs1801265 and rs67376798 of the DPYD gene, rs 3758149 of the GGH gene, and rs1042927 of the RRM1 gene. These markers were thus excluded from the remaining statistical analyses. 


\begin{tabular}{|c|c|c|c|c|c|c|c|c|c|c|}
\hline \multirow[b]{2}{*}{ No. } & \multirow[b]{2}{*}{ Reference SNP ID } & \multirow[b]{2}{*}{ Gene Definig SNP } & \multirow[b]{2}{*}{$\mathrm{ALL}^{\mathrm{a}}$} & \multirow[b]{2}{*}{ AFR } & \multicolumn{2}{|c|}{$\begin{array}{l}\text { Allele } \\
\text { Frequencies }\end{array}$} & \multirow[b]{2}{*}{ EUR } & \multirow[b]{2}{*}{ SAS } & \multicolumn{2}{|c|}{$\begin{array}{l}\text { Hardy-Weinberg } \\
\text { Equilibrium (P-Value) }\end{array}$} \\
\hline & & & & & AMR & EAS & & & IND & \\
\hline 1 & rs1045642 & $A B C B 1$ & 0,40 & 0.15 & 0.43 & 0.4 & 0.52 & 0.57 & 0,50 & 0.229 \\
\hline 2 & rs1128503 & $A B C B 1$ & 0,42 & 0.14 & 0.4 & 0.63 & 0.42 & 0.59 & 0,51 & 1.000 \\
\hline 3 & rs717620 & $A B C C 2$ & 0,13 & 0.03 & 0.17 & 0.22 & 0.21 & 0.1 & 0,11 & 0.003 \\
\hline 4 & rs4148551 & $A B C C 4$ & 0,49 & 0.59 & 0.43 & 0.5 & 0.38 & 0.5 & 0,54 & 0.175 \\
\hline 5 & rs3742106 & $A B C C 4$ & 0,41 & 0.31 & 0.41 & 0.5 & 0.38 & 0.5 & 0,52 & \begin{tabular}{|l|}
0.437 \\
\end{tabular} \\
\hline 6 & rs9524885 & $A B C C 4$ & 0,41 & 0.63 & 0.29 & 0.42 & 0.27 & 0.36 & 0,2 & 4.80E-04 \\
\hline 7 & rs2231142 & $A B C G 2$ & 0,12 & 0.01 & 0.14 & 0.29 & 0.09 & 0.1 & 0,43 & 0.475 \\
\hline 8 & rs28399433 & CYP2A6 & 0,13 & 0.08 & 0.1 & 0.24 & 0.07 & 0.15 & 0,46 & 0.600 \\
\hline 9 & rs8192726 & CYP2A6 & 0,9 & 0.92 & 0.96 & 0.82 & 0.93 & 0.87 & 0,74 & 0.00E-00 \\
\hline 10 & rs17116806 & DPYD & 0,17 & 0.1 & 0.26 & 0.27 & 0.19 & 0.08 & 0,52 & 0.684 \\
\hline 11 & rs1760217 & DPYD & 0,2 & \begin{tabular}{|l|}
0.19 \\
\end{tabular} & 18 & 0.3 & \begin{tabular}{|l|}
0.18 \\
\end{tabular} & 0.15 & 0,19 & 1.000 \\
\hline 12 & rs 1801159 & DPYD & 0,18 & 0.15 & 0.27 & 0.27 & 0.19 & 0.08 & 0,45 & \begin{tabular}{|l|l|}
0.714 \\
\end{tabular} \\
\hline 13 & rs1801265 & DPYD & 0,74 & 0.56 & 0.78 & 0.91 & \begin{tabular}{|l|}
0.79 \\
\end{tabular} & 0.73 & 0,79 & \begin{tabular}{|l|}
$1.33 \mathrm{E}-03$ \\
\end{tabular} \\
\hline 14 & rs3918290 & DPYD & 0 & 0 & 0 & - & 0 & 0.01 & 0,01 & 1.000 \\
\hline 15 & rs4970722 & DPYD & 0,22 & 0.29 & 0.22 & 0.09 & 0.21 & 0.26 & 0,45 & 0.013 \\
\hline 16 & rs55886062 & DPYD & 0 & - & - & - & 0 & - & 0,02 & 1.000 \\
\hline 17 & rs67376798 & DPYD & 0 & 0 & 0 & - & 0.01 & 0 & 0,04 & 3.60E-04 \\
\hline 18 & rs17376848 & DPYD & 0,05 & 0.01 & 0.08 & 0.11 & 0.04 & 0.03 & 0,27 & 0.251 \\
\hline 19 & rs4451422 & FPGS & 0,54 & 0.6 & 0.54 & 0.31 & 0.61 & 0.6 & 0,58 & 0.152 \\
\hline 20 & rs3758149 & GGH & 0,23 & \begin{tabular}{|l|l|}
0.17 \\
\end{tabular} & 0.23 & 0.22 & 0.28 & 0.29 & 0,60 & \begin{tabular}{|l|}
$1.6 \mathrm{E}-04$ \\
\end{tabular} \\
\hline 21 & rs10049380 & ITGB5 & 0,66 & 0.54 & 0.61 & 0.68 & 0.81 & 0.68 & 0,30 & 0.217 \\
\hline 22 & rs 1801131 & MTHFR & 0,25 & 0.15 & 0.15 & 0.22 & 0.31 & 0.42 & 0,09 & 0.002 \\
\hline 23 & rs 1801133 & MTHFR & 0,25 & 0.09 & 0.47 & 0.3 & 0.36 & 0.12 & 0,23 & 0.006 \\
\hline 24 & rs1042927 & RRMI & 0,85 & 0.8 & 0.83 & 0.76 & 0.93 & 0.93 & 0,80 & \begin{tabular}{|l|}
$1.37 \mathrm{E}-03$ \\
\end{tabular} \\
\hline 25 & rs12806698 & RRM1 & 0,23 & 0.03 & 0.22 & 0.3 & 0.28 & 0.36 & 0,42 & 0.327 \\
\hline 26 & rs2270860 & SLC22A7 & 0,46 & 0.72 & 0.38 & 0.37 & 0.34 & 0.4 & 0,43 & 0.850 \\
\hline 27 & rs4149178 & SLC22A7 & 0,19 & 0.34 & 0.21 & 0.05 & \begin{tabular}{|l|}
0.17 \\
\end{tabular} & 0.13 & 0,36 & 0.202 \\
\hline 28 & \begin{tabular}{|l|} 
rs747199 \\
\end{tabular} & SLC29A1 & 0,15 & 0.01 & 0.18 & 0.27 & 0.22 & 0.1 & 0,64 & 0.006 \\
\hline 29 & \begin{tabular}{|l|} 
rs760370 \\
\end{tabular} & SLC29A1 & 0,3 & 0.26 & 0.37 & 0.3 & 0.4 & 0.2 & 0,40 & 0.690 \\
\hline 30 & rs 1042522 & TP53 & 0,54 & 0.33 & 0.68 & 0.59 & \begin{tabular}{|l|}
0.71 \\
\end{tabular} & 0.51 & 0,73 & 0.073 \\
\hline 31 & rs11479 & TYMP & 0,14 & 0.03 & 0.17 & 0.29 & \begin{tabular}{|l|}
0.07 \\
\end{tabular} & 0.19 & 0,26 & 0.453 \\
\hline 32 & rs1801019 & UMPS & 0,19 & \begin{tabular}{|l|}
0.15 \\
\end{tabular} & 0.26 & 0.17 & 0.15 & 0.23 & 0,54 & 0.324 \\
\hline
\end{tabular}

Table 1. Hardy Weinberg Equilibrium and Comparison of allelic frequencies of ADME-related SNPs in Amerindian population (IND) and others continental populations (AFR, AMR, EAS, EUR, SAS). ${ }^{a}$ ALL represents the mean values of the five continental populations (AFR, AMR, EAS, EUR and SAS).

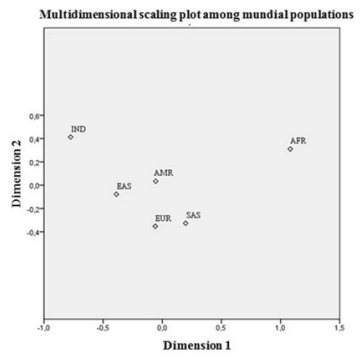

Figure 1. Multidimensional scaling plot illustrating the grouping of ethnic populations according to the genetic profile of the 26 ADME-markers.

The multidimensional scaling (MDS) analysis of the $\mathrm{F}_{\mathrm{ST}}$ values for each pairwise comparison revealed the similarities and differences in the 26 SNPs related to the ADME drug process (Fig. 1; Table 2). The graph highlights population clusters, which indicate a very distinct genetic profile between the Amerindian and African populations, which are isolated at the extremities of the plot. The genetic profile of the Amerindian population is most similar to those of the American and East Asian populations. 


\begin{tabular}{|l|l|l|l|l|l|l|}
\hline & AFR & AMR & EAS & EUR & IND & SAS \\
\hline AFR & - & & & & & \\
\hline AMR & 0.09588 & - & & & & \\
\hline EAS & 0.13062 & 0.03255 & - & & & \\
\hline EUR & 0.11534 & 0.01477 & 0.04566 & - & & \\
\hline IND & 0.18313 & 0.06042 & 0.05831 & 0.09851 & - & \\
\hline SAS & 0.10250 & 0.04414 & 0.04690 & 0.03268 & 0.09548 & - \\
\hline
\end{tabular}

Table 2. Pairwise FST among Amerindians and the five continental populations from 1000 genomes database.

\begin{tabular}{|c|c|c|c|c|c|c|c|c|}
\hline \multirow[b]{2}{*}{ No. } & \multirow[b]{2}{*}{$\begin{array}{l}\text { Reference } \\
\text { SNP ID }\end{array}$} & \multirow[b]{2}{*}{\begin{tabular}{|l|} 
Gene \\
Defining SNP
\end{tabular}} & \multirow[b]{2}{*}{$\begin{array}{l}\text { IND versus } \\
\text { ALL }\end{array}$} & \multirow[b]{2}{*}{$\begin{array}{l}\text { IND versus } \\
\text { AFR }\end{array}$} & \multicolumn{2}{|c|}{$\begin{array}{l}\text { Pairwise Comparison } \\
\text { (P-Value) }\end{array}$} & \multirow[b]{2}{*}{$\begin{array}{l}\text { IND versus } \\
\text { EUR }\end{array}$} & \multirow[b]{2}{*}{$\begin{array}{l}\text { IND versus } \\
\text { SAS }\end{array}$} \\
\hline & & & & & $\begin{array}{l}\text { IND versus } \\
\text { AMR }\end{array}$ & $\begin{array}{l}\text { IND versus } \\
\text { EAS }\end{array}$ & & \\
\hline 1 & rs 1045642 & $A B C B 1$ & 0,000351 & $8,569 \mathrm{E}-034$ & 0,087509 & 0,004795 & 0,459556 & 0,024838 \\
\hline 2 & rs1128503 & $A B C B 1$ & 0,010039 & 5,748E-039 & 0,012830 & 0,003571 & 0,459556 & 0,082449 \\
\hline 3 & rs717620 & $A B C C 2$ & 0,029090 & 3,565E-007 & 0,001932 & 0,000087 & 0,000010 & 0,059195 \\
\hline 4 & rs4148551 & $A B C C 4$ & 0,217774 & 0,117681 & 0,012613 & 0,375018 & \begin{tabular}{|l|l|}
0,000090 \\
\end{tabular} & \begin{tabular}{|l|}
0,177281 \\
\end{tabular} \\
\hline 5 & rs3742106 & $A B C C 4$ & 0,004675 & 4,441E-009 & 0,014532 & 0,732544 & 0,000521 & 0,468344 \\
\hline 6 & rs2231142 & ABCG2 & 2,336E-044 & $1,045 \mathrm{E}-085$ & 7,047E-019 & \begin{tabular}{|l|l|}
0,000050 \\
\end{tabular} & $4,795 \mathrm{E}-032$ & $3,619 \mathrm{E}-033$ \\
\hline 7 & rs28399433 & CYP2A6 & 3,368E-042 & 7,608E-048 & $4,113 \mathrm{E}-031$ & $1,402 \mathrm{E}-011$ & $1,901 \mathrm{E}-046$ & $1,975 \mathrm{E}-022$ \\
\hline 8 & rs17116806 & $D P Y D$ & 1,669E-033 & 6,134E-046 & $5,005 \mathrm{E}-010$ & $2,948 \mathrm{E}-010$ & $1,522 \mathrm{E}-019$ & $1,035 \mathrm{E}-043$ \\
\hline 9 & rs1760217 & DPYD & 0,833815 & 0,995618 & 0,953805 & 0,004403 & 0,912777 & 0,395147 \\
\hline 10 & rs1801159 & DPYD & 2,491E-021 & $8,065 \mathrm{E}-024$ & 0,000002 & 3,785E-007 & 2,730E-015 & $1,776 \mathrm{E}-038$ \\
\hline 11 & rs3918290 & DPYD & 0,002800 & 0,003626 & 0,024202 & 0,002015 & 0,103855 & 0,328929 \\
\hline 12 & rs4970722 & DPYD & 5,305E-017 & 2,348E-007 & 3,392E-012 & $1,015 \mathrm{E}-041$ & 2,247E-014 & 1,952E-009 \\
\hline 13 & rs55886062 & DPYD & 0,000002 & \begin{tabular}{|l|l|}
0,000149 \\
\end{tabular} & 0,001817 & 0,000446 & 0,002224 & \begin{tabular}{|l|l|}
0,00502 \\
\end{tabular} \\
\hline 14 & rs17376848 & DPYD & $3,229 \mathrm{E}-043$ & $2,087 \mathrm{E}-048$ & $9,420 \mathrm{E}-012$ & $3,681 \mathrm{E}-009$ & $4,826 \mathrm{E}-027$ & $1,008 \mathrm{E}-026$ \\
\hline 15 & rs4451422 & FPGS & \begin{tabular}{|l|}
0,379278 \\
\end{tabular} & 0,815190 & 0,347094 & $4,991 \mathrm{E}-015$ & 0,230975 & 0,256130 \\
\hline 16 & rs10049380 & ITGB5 & $1,651 \mathrm{E}-026$ & 1,252E-009 & $1,307 \mathrm{E}-012$ & $7,000 \mathrm{E}-024$ & 4,073E-051 & 7,416E-021 \\
\hline 17 & rs1801131 & MTHFR & \begin{tabular}{|c|}
$1,016 \mathrm{E}-009$ \\
\end{tabular} & 0,001766 & 0,001990 & $1,000 \mathrm{E}-007$ & $4,887 \mathrm{E}-014$ & 4,301E-025 \\
\hline 18 & rs1801133 & MTHFR & \begin{tabular}{|l|l|}
0,299989 \\
\end{tabular} & 3,303E-010 & $3,500 \mathrm{E}-014$ & 0,021084 & 0,000004 & $1,033 \mathrm{E}-007$ \\
\hline 19 & rs12806698 & RRM1 & $5,917 \mathrm{E}-010$ & 4,437E-059 & $2,146 \mathrm{E}-008$ & 0,001617 & \begin{tabular}{|l|l|}
0,000164 \\
\end{tabular} & 0,069652 \\
\hline 20 & rs2270860 & SLC22A7 & 0,495893 & 2,488E-017 & 0,355337 & 0,180118 & 0,042493 & 0,563101 \\
\hline 21 & rs4149178 & SLC22A7 & $3,976 \mathrm{E}-008$ & 0,593274 & 0,000123 & $1,956 \mathrm{E}-032$ & $1,975 \mathrm{E}-009$ & 9,646E-015 \\
\hline 22 & \begin{tabular}{|l|l|} 
rs747199 \\
\end{tabular} & SLC29A1 & 1,111E-020 & \begin{tabular}{|l|}
$1,000 \mathrm{E}-069$ \\
\end{tabular} & 9,135E-009 & 0,000141 & 7,604E-007 & $1,067 \mathrm{E}-018$ \\
\hline 23 & rs760370 & SLC29A1 & 0,011188 & 0,000199 & \begin{tabular}{|l}
0,799858 \\
\end{tabular} & 0,012188 & 0,965590 & 8,367E-009 \\
\hline 24 & rs1042522 & TP53 & \begin{tabular}{|l|l|}
$488 E-007$ \\
\end{tabular} & 4,528E-029 & 0,103918 & 0,000133 & 0,115445 & 6,061E-009 \\
\hline 25 & rs11479 & TYMP & 0,000019 & 6,933E-032 & 0,011643 & 0,582035 & $4,960 \mathrm{E}-014$ & 0,025776 \\
\hline 26 & rs1801019 & UMPS & \begin{tabular}{|c|}
$5,623 \mathrm{E}-040$ \\
\end{tabular} & 1,341E-037 & 5,362E-012 & 5,505E-026 & $1,492 \mathrm{E}-034$ & $8,120 \mathrm{E}-016$ \\
\hline
\end{tabular}

Table 3. Pairwise comparison of genotypic frequencies in Amerindians with each one of the five continental populations from 1000 genomes database.

The 26 SNPs were also analyzed in pairwise comparisons between the IND population and the mean values for the five 1000 Genomes populations (ALL), as well as each of these populations individually, in order to characterize the unique aspects of the genetic profile of the Amerindian population, in comparison with those of the other populations (Table 3 ).

In the comparison between the IND and the AFR populations, the distribution of 20 polymorphisms of 14 genes was significantly different between populations. Eight of the polymorphisms involved six transporter genes: the $A B C B 1, A B C C 2, A B C C 4, A B C G 2, S L C 22 A 7$, and SLC29A1 genes. In the case of the phase I genes, there were two groups, the CYP2A6 and the DPYD genes with six significantly different polymorphisms. We also observed significantly different distributions in six polymorphisms in six other genes involved in the ADME processing of drug: the ITGB5, MTHFR, RRM1, TP53, TYMP and UMPS genes.

By contrast, we identified only 12 polymorphisms of nine genes that varied significantly between the IND and the AMR populations. These included one polymorphism in each of the three transporter genes: $A B C G 2$, $S L C 22 A 7$ and SLC29A1. Five polymorphisms were found in the phase I genes, CYP2A6 and DPYD. Four polymorphisms of four other ADME genes were also significantly different, that is, the ITGB5, MTHFR, RRM1 and UMPS genes. 
In the comparison between the IND and the EAS populations, 16 polymorphisms of 11 genes had a significantly different distribution. These included five polymorphisms of four transporter genes: the $A B C C 2, A B C G 2$, $S L C 22 A 7$, and SLC29A1 genes. Significant differences were also found in the two phase I genes, CYP2A6 and $D P Y D$. A significant level of variation was also found in a further five polymorphisms, of five genes, the FPGS, ITGB5, MTHFR, TP53 and UMPS.

Pairwise comparisons between the IND and the EUR populations revealed significant differences in 18 polymorphisms in 12 genes. Six of these polymorphisms involved five transporter genes: $A B C C 2, A B C C 4, \mathrm{ABCG}$, $S L C 22 A 7$ and SLC29A1. In the phase I genes, significant differences were found in one polymorphism of the CYP2A6 gene and in four polymorphisms of the DPYD gene. The analysis also demonstrated significant differences in seven polymorphisms in five other genes related to the ADME: the ITGB5, MTHFR, RRM1, TYMP, and UMPS genes.

Finally, significant differences were identified between the IND and SAS populations in 16 polymorphisms present in 10 genes. Five of these polymorphisms were present in four transporter genes: $A B C B 1, \mathrm{ABCG}$, $S L C 22 A 7$, and SLC29A1. Six of the variants were also found in the phase I genes, CYP2A6 and DPYD. The five other markers were identified in four other ADME-related genes, ITGB5, MTHFR, TP53 and UMPS.

\section{Discussion}

Adverse Drug Reactions (ADRs) are one of the principal causes of morbidity in developed countries ${ }^{19}$. High rates of hospitalization due to ADRs are caused, in part, by the standardization of drug doses, which overlooks the variability among patients in factors such as the symptoms of the disease, environmental and genetic characteristics, and ethnicity ${ }^{20,21}$.

Another important factor to be considered during treatment choice is drug efficacy, the ability of a drug to achieve the desired effect ${ }^{22}$. The main challenge to achieve maximum effectiveness of a drug is also the interindividual variability in drug response. Drug mechanisms focused on targets with genetic support would succeed twice as often as those without it, reducing the clinical trial's phases due to lower rates of failure caused by lack of efficacy during clinical development ${ }^{23}$.

Most pharmacogenetic studies focus on Caucasian populations, which cannot necessarily be extrapolated reliably to the application of PGx in other ethnic groups, such as Amerindian, which may have a unique genetic profile resulting from prolonged geographic isolation and inbreeding ${ }^{24,25}$. There have been relatively few studies of molecular biomarkers in Amerindian populations, highlighted by the fact that they are absent from the 1000 Genomes database, the largest available collection of data on human genetic variability.

This emphasizes the need for the investigation in other ethnic groups, in particular Amerindians, of predictive molecular biomarkers known to play a role in specific treatments or other, as yet undiscovered markers, in order to understand the genetic variability of these populations and to use the knowledge to delineate personalized treatment protocols, in order to improve drug security in Amerindian populations from the Amazon region, as well as in other populations with a high degree of admixture with this ethnic group.

In the present study, we compared the genetic variability of Amerindian populations from the Amazon region with five populations from the 1000 Genomes Project, analyzing 26 genes responsible for the absorption, distribution, metabolization, and excretion of drugs. The $\mathrm{F}_{\mathrm{ST}}$ indices indicate that the Amerindian and African populations were the most distinct genetically, which is consistent with the history of the world's human populations, in which the Amerindian and African groups represent the extremes of the evolutionary process ${ }^{26}$.

Still regarding the $\mathrm{F}_{\mathrm{ST}}$ analysis, the lower values of genetic differences with the Amazonian Amerindians is observed on the East Asian population $\left(\mathrm{F}_{\mathrm{ST}}\right.$ value $\left.=0.05831\right)$, which is mainly composed of Chinese and Japanese individuals. This outcome corroborates with the "Bering Strait" hypothesis, which discusses that the settlement of the Americas and the formation of the first Native Americans are due the migrations of Asians populations that occurred less than 15,000 years ago through a land extension known as the Bering land bridge, which joined Northeast Asia and North America ${ }^{27}$.

Relying up on the $\mathrm{F}_{\mathrm{ST}}$ analysis concomitant with the pairwise comparisons analysis, the Amerindian population is genetically most similar to the American population, with significant differences being found in only 12 polymorphisms, and an $\mathrm{F}_{\mathrm{ST}}$ value of 0.06042 . The American samples from the 1000 Genomes database includes Colombian (CLM), Puerto Rican (PUR), Peruvian individuals (PEL), and Californian residents of Mexican descent (MXL), who are representative of the different populations of Latin America, which implies a certain degree of genetic similarity with the ancestral populations of South and Central America ${ }^{28}$.

In fact, high levels of interethnic admixture have already been shown in populations from the Americas, and in particular, from Latin America ${ }^{29}$. This genetic admixture originated during the European colonization of the New World, when native Americans came into contact with European immigrants arriving on the continent from 1492 onward, and with African immigrants, from 1502 onward $^{30}$.

Gravel et al. (2012) investigated the exome of the Colombian, Mexican and Puerto Rican populations from the 1000 Genomes database and estimated the contributions of African, European, and Native American ancestors to these populations. The findings of the study indicated that Amerindians contributed $12.8 \%$ of the PUR exome, $25.6 \%$ of that from CLM, and $47.6 \%$ from $\mathrm{MXL}^{31}$. There are still no studies that evaluate the ancestry of Peruvian samples in the 1000 Genomes database, however, Homburger et al. (2015) evaluated 119 individuals from Peru and determined that they had an Amerindian ancestry of $68.3 \%{ }^{32}$.

The profile of the genomic ancestry of Latin American countries thus indicates considerable heterogeneity in terms of the contribution of Amerindians, resulting from the varied history of the formation of these populations, and their degree of interethnic admixture. The study of ADME polymorphisms in native American populations is, therefore, fundamentally importance to ensure the optimal implementation of healthcare programs that include genomic information on both Latin and Amerindian populations. 
Here, we discuss specifically the data on three of the genes - ABCB1, CYP2A6 and DPYD- whose profiles in the Amerindian population were significantly different from the other study populations. The impact on the enzyme activity caused by the allelic variants analysed is described at Supplementary Table 1 (Table SI). These genes are widely cited as pharmacogenetic biomarkers capable of significant alterations in the ADME patterns of a number of different classes of drugs, with their clinical significance being recognized in the pharmacogenetic labelling of a number of different medications by prominent drug regulatory agencies, including the American Food and Drug Administration (FDA), the European Medicines Agency (EMA), the Japanese Pharmaceuticals and Medical Devices Agency (PMDA), and the Canadian Health Canada Santé Canada (HCSC) ${ }^{33}$.

$A B C B 1$ and Aliskiren. The $A B C B 1$ gene (P-glycoprotein encoding [P-gp]) belongs to the ATP-binding cassete $(\mathrm{ABC})$ superfamily of human carriers, and plays a key role in the absorption, distribution, and elimination of drugs within the organism, being considered one of the major determinants of drug resistance ${ }^{34}$. The rs 1128503 and rs1045642 polymorphisms of the $A B C B 1$ gene have been identified as have a potential role in the response to and toxicity of a number of different drugs, including imatinib, opioids and treatments for epilepsy ${ }^{35-37}$.

The European and Canadian drug regulatory authorities require the inclusion of pharmacokinetic information on their drug labels on the interaction between the $A B C B 1$ gene and Aliskiren (Rasilez $\mathrm{HCT}^{\circledR}$ ), an antihypertensive drug. The EMA identifies $A B C B 1$ as the principal efflux system involved in the intestinal absorption and biliary excretion of the drug and contraindicates the concomitant administration of Aliskiren and medications that may influence $A B C B 1$ gene action ${ }^{38}$. The HCSC also refers to the role of the $A B C B 1$ gene in the Aliskiren efflux system in its drug labelling, contraindicating the use of different glycoprotein inhibitors and other drugs during treatment with Aliskiren ${ }^{39}$. Polymorphisms on the $A B C B 1$ gene also may cause a polymorphic enzyme of $\mathrm{P}$-gp with diminished activity ${ }^{40}$, therefore, should also be considered as an influence factor of Aliskiren efficacy.

In the present study, we found high allele frequencies of the $A B C B 1$ variants (of at least $40 \%$ ) in the Amerindian populations from the Amazon Region. These variants may reduce glycoprotein activity and thus alter the metabolization of antihypertensive drugs, such as Aliskiren, affecting its efficacy in the treatment of Amerindian populations.

CYP2A6 and Letrozole. The cluster of CYP2 genes on chromossome 19 includes six families, one of which is the CYP2A family, with three genes, the CYP2A6, CYP2A7, and CYP2A13. The CYP2A6 gene is highly polymorphic, and its protein product represents one of the principal liver enzymes responsible for the metabolism of drugs and xenobiotics ${ }^{41}$.

The Japanese Pharmaceutical Regulatory Agency (PMDA) includes pharmacogenetic information on the CYP2A6 gene on its Letrozole label. Letrozole is a chemotherapeutic drug used to treat breast cancer. In vitro studies have shown that it is metabolized primarily in its inactive form in liver microsomes through the action of the CYP2A6 gene ${ }^{42}$.

Patients identified as poor or normal metabolizers based on their CYP2A6 polymorphisms, which modify enzyme activity, may suffer either an increased toxicity profile or treatment shortcomings. There is a wide range of functional activity data for the described variants of CYP2A6, leading to either differences in enzyme activity or mRNA expression and, consequently, protein levels ${ }^{43}$. Patients with specific polymorphic variants or a combination of CYP2A6 polymorphisms that define them as poor metabolizers may present a twofold increase in their plasmatic concentrations of Letrozole when compared with extensive metabolizers ${ }^{42}$.

One of the markers associated with the poor metabolizer profile is CYP2A6*9 (rs28399433), which was extremely frequent (46\%) in the Amerindian population, and much higher than in the other populations, in which the mean frequency is $13 \%$. This indicates that the Amerindian population from the Amazon region has an abnormally high proportion of poor metabolizers, due to the frequency of the CYP $2 A 6 * 9$ variant, which may determine adverse toxic reactions during treatment with Letrozole, and other drugs that are metabolized by the CYP2A subfamily.

DPYD and Capecitabine + Fluorouracil. The DPYD gene is composed of 23 exons at the $1 \mathrm{p} 22$ chromosomal locus. This gene encodes the enzyme dihydropyrimidine dehydrogenase (DPD), which accounts for about $85 \%$ of the hepatic catabolism of 5-fluorouracil to its inactive metabolic form, 5- fluoro-5,6-dihydrofluorouracil ${ }^{44}$. Because this is an essential enzyme in the metabolism of 5-fluorouracil, patients with low DPD enzyme activity have a higher risk of developing severe and even lethal toxicities during standard chemotherapeutic treatment with this drug ${ }^{45}$.

The guidelines of the Clinical Pharmacogenetics Implementation Consortium (CPIC) indicate three DPYD genotypes as the major non-functional variants of this gene: *2 A (rs3918290), *13 (rs55886062), and rs67376798. The CPIC guidelines characterize phenotypes based on each of these three variants, and strongly recommend the use of alternative drugs or the reduction of the standard 5-Fluorouracil dose by $50 \%$ for patients who are either homozygous or heterozygous for any of these three variants ${ }^{46}$.

A number of national drug regulatory authorities include information on the DPYD gene in their pharmacogenetic drug labels. Capecitabine is one of the major 5- Fluorouracil prodrugs used to treatment various types of solid neoplasias. The FDA includes a warning note in its label for this drug (XELODA ${ }^{\circledR}$ ), advising patients who have genotypes associated with an absence or reduced activity of DPD that they are at a higher risk of developing severe or potentially lethal adverse reactions ${ }^{47}$.

The FDA also includes pharmacogenetic information labels on the potential for severe toxicity in patients with DPD deficiency during treatment with Fluorouracil, describing the different types of adverse reaction presented by patients with homozygous or heterozygous mutations resulting from a partial or total deficiency of the DPD enzyme. The FDA recommends discontinuing permanently the use of Fluorouracil based on the severity of the toxicities observed in patients treated by the $5-\mathrm{FU}$ regime ${ }^{48,49}$. 
Nine polymorphic variants of the DPYD gene were identified in our Amerindian population. The allele frequencies of the rs17116806, rs 1801159 and rs4970722 markers were all higher than 40\%, contrasting with the much lower frequencies found in all other populations around the world. Two of three deleterious polymorphisms mentioned in the CPIC guidelines were identified in our Amerindian population. The frequency of the rs3918290 marker was 1\%, similar to that of most other populations, in particular those from Asia. The rs55886062 marker had a frequency of $2 \%$, however, which is relatively high in comparison with the other populations, in which it did not exceed $1 \%$, when present.

In our Amerindian population, the frequency of the rs 1801133 mutation was consistent with that found in the other study populations (mean $=23 \%$ ), whereas the rs 1801131 polymorphism had a low frequency $(9 \%)$ compared to the other populations (mean $=25 \%$ ). This indicates a lower risk of developing serious adverse effects in Amerindian women, associated with the use of contraceptives based on norelgestromin and ethinylestradiol.

Up until now, there has been no systematic assessment of the potential response of Amerindian ethnic groups from the Amazon basin to treatment with drugs known to be influenced by genetic polymorphisms. Our study evaluated the unique features in the genetic profile of 26 ADME-related genes in an Amazonian Amerindian population comparing with others continental populations, which may reflect a distinct therapeutic profile in relation to the efficacy and toxicity of drugs marketed widely for the treatment of a range of diseases. Given this, there is a clear need for the understanding of the individual genetic profiles of Amerindians, and the application of this knowledge to the design of specific pharmacogenomic-guided treatment protocols for indigenous Amazon ethnic groups. This approach would also be valid for populations, such as that of Brazil, which present a high degree of miscegenation with this indigenous group. As future perspectives of our research group regarding this study, data on other clinically better known ADME polymorphisms will further be reported in a separate paper.

\section{References}

1. Nelson, M. R. et al. The genetics of drug efficacy: opportunities and challenges. Nat Rev Genet. 17, 197-206 (2016).

2. Ramos, E. et al. Pharmacogenomics, ancestry and clinical decision making for global populations. Pharmacogenomics J. 14, 217-22 (2014).

3. Jittikoon, J. et al. Comparison of genetic variation in drug ADME-related genes in Thais with Caucasian, African and Asian HapMap populations. J Hum Genet. 61, 119-27 (2016).

4. Gamazon, E. R. \& Perera, M. Genome-wide approaches in pharmacogenomics: heritability estimation and pharmacoethnicity as primary challenges. Pharmacogenomics. 13, 1101-4 (2012).

5. O'Donnell, P. H. \& Dolan, M. E. Cancer pharmacoethnicity: ethnic differences in susceptibility to the effects of chemotherapy. Clin Cancer Res. 15, 4806-14 (2009).

6. Ortega, V. E. \& Meyers, D. A. Pharmacogenetics: implications of race and ethnicity on defining genetic profiles for personalized medicine. J Allergy Clin Immunol. 133, 16-26 (2014).

7. Yasuda, S., Zhang, L. \& Huang, S. M. The role of ethnicity in variability in response to drugs: focus on clinical pharmacology studies. Clin Pharmacol Ther. 84, 417-23 (2008).

8. Scott, S. A. Personalizing medicine with clinical pharmacogenetics. Genet Med. 13, 987-95 (2011).

9. Li, J., Zhang, L., Zhou, H., Stoneking, M. \& Tang, K. Global patterns of genetic diversity and signals of natural selection for human ADME genes. Hum Mol Genet. 20, 528-40 (2011).

10. Santos, N. P. C. et al. Assessing individual interethnic admixture and population substructure using a 48-insertion-deletion (INSEL) ancestry-informative marker (AIM) panel. Hum Mutat. 31, 184-90 (2010).

11. Suarez-Kurtz, G. Pharmacogenetics in the Brazilian population. Front Pharmacol. 1, 1-10 (2010).

12. Cerda, A., Hirata, M. H. \& Hirata, R. D. C. Pharmacogenetics of drug metabolizing enzymes in Brazilian populations. Drug Metabol Drug Interact. 29, 153-77 (2014).

13. Rodrigues-Soares, F. et al. Genetic structure of pharmacogenetic biomarkers in Brazil inferred from a systematic review and population-based cohorts: a RIBEF/EPIGEN-Brazil initiative. Pharmacogenomics J. (Epub ahead of print) (2018).

14. Instituto Brasileiro de Geografia e Estatística (IBGE), https://indigenas.ibge.gov.br/images/pdf/indigenas/folder_indigenas_web. pdf.

15. Lindenau, J. D. et al. Distribution patterns of variability for 18 immune system genes in Amerindians - relationship with history and epidemiology. Tissue Antigens. 82, 177-85 (2013).

16. Lindenau, J. D. R. et al. Variability of innate immune system genes in Native American populations - Relationship with history and epidemiology. Am J Phys Anthropol. 159, 722-8 (2016)

17. Mychaleckyj, J. C. et al. Genome-wide analysis in brazilians reveals highly differentiated native American genome regions. Mol Biol Evol. 34, 559-74 (2017)

18. Excoffier, L. \& Lischer, H. E. L. Arlequin suite ver 3.5: A new series of programs to perform population genetics analyses under Linux and Windows. Mol Ecol Resour. 10, 564-7 (2010).

19. Maroñas, O. et al. Progress in pharmacogenetics: consortiums and new strategies. Drug Metab Pers Ther. 31, 17-23 (2016).

20. Pirmohamed, M. Personalized pharmacogenomics: predicting efficacy and adverse drug reactions. Annu Rev Genomics Hum Genet. 15, 349-70 (2014).

21. Verbelen, M., Weale, M. E. \& Lewis, C. M. Cost-effectiveness of pharmacogenetic-guided treatment: are we there yet? Pharmacogenomics J. 17, 395-402 (2017).

22. Nelson, M. R. et al. The genetics of drug efficacy: opportunities and challenges. Nat. Rev. Genet. 17, 197-206 (2016).

23. Nelson, M. R. et al. The support of human genetic evidence for approved drug indications. Nat. Genet. 47, 856-860 (2015).

24. Suarez-Kurtz, G., Paula, D. P. \& Struchiner, C. J. Pharmacogenomic implications of population admixture: Brazil as a model case. Pharmacogenomics. 15, 209-19 (2014).

25. Ruiz-Linarés, A. How genes have illuminated the history of early americans and latino americans. Cold Spring Harb Perspect Biol. 7 , 1-9 (2015).

26. Skoglund, P. \& Reich, D. A genomic view of the peopling of the Americas. Curr Opin Genet Dev. 41, 27-35 (2016).

27. Hoffecker, J. F., Elias, S. A., O’Rourke, D. H., Scott, G. R. \& Bigelow, N. H. Beringia and the global dispersal of modern humans. Evol. Anthropol. 25, 64-78 (2016)

28. Wang, S. et al. Genetic variation and population structure in Native Americans. PLoS Genet. 11, 2049-67 (2007).

29. Reich, D. et al. Reconstructing Native American population history. Nature. 488, 370-4 (2012).

30. Adhikari, K., Mendoza-Revilla, J., Chacón-Duque, J. C., Fuentes-Guajardo, M. \& Ruiz-Linares, A. Admixture in Latin America. Curr Opin Genet Dev. 41, 106-14 (2016).

31. Gravel, S. et al. Reconstructing Native American migrations from whole-genome and whole-exome data. PLoS Genet. 9, e1004023 (2013).

32. Homburger, J. R. et al. Genomic insights into the ancestry and demographic history of South America. PLoS Genet. 11, 1-26 (2015). 
33. Whirl-Carrillo, M. et al. Pharmacogenomics knowledge for personalized medicine. Clin Pharmacol Ther. 92, 414-7 (2012).

34. Bruhn, O. \& Cascorbi, I. Polymorphisms of the drug transporters ABCB1, ABCG2, ABCC2 and ABCC3 and their impact on drug bioavailability and clinical relevance. Expert Opin Drug Metab Toxicol. 10, 1337-54 (2014).

35. Zheng, Q. et al. ABCB1 polymorphisms predict imatinib response in chronic myeloid leukemia patients: a systematic review and meta-analysis. Pharmacogenomics J. 15, 127-34 (2015).

36. Zahari, Z. et al. Relationship between ABCB1 polymorphisms and cold pain sensitivity among healthy opioid-naive Malay males. Pain Pract. 17, 930-40 (2017).

37. Sun, F., Cao, B. Q., Wang, B., Wu, S. Q. \& Jiang, D. H. Association between ABCB1 genetic polymorphism and the effect on epilepsy following phenytoin treatment. Exp Ther Med. 12, 1780-4 (2016).

38. Rasilez, H. C. T. ${ }^{\circledR}$ (Aliskiren) [package insert on the internet]. Italy: Novartis Farma S.p.A.; [revised 2014 Jan]. Available from: http://www.ema.europa.eu/docs/en_GB/document_library/EPAR_Product_Information/human/000964/WC500047220.pdf (2009).

39. Rasilez, H. C. T. ${ }^{\circledR}$ (Aliskiren) [package insert on the internet]. Dorval: Novartis Pharmaceuticals Canada Inc.; Available from: https://pdf.hres.ca/dpd_pm/00035145.PDF [May 31, 2016].

40. Vaalburg, W., Hendrikse, N. H., Elsinga, P. H., Bart, J. \& Van Waarde, A. P-glycoprotein activity and biological response. Toxicol Appl Pharmacol. 207, 257-260 (2005).

41. Raunio, H. \& Rahnasto-Rilla, M. CYP2A6: Genetics, structure, regulation, and function. Drug Metabol Drug Interact. 27, 73-88 (2012).

42. Letrozole [package insert on the internet]. Japan: Novartis. Available from: https://www.pharmgkb.org/label/PA166160717.

43. Tanner, J. A. \& Tyndale, R. Variation in CYP2A6 activity and personalized medicine. J Pers Med. 7, 18-29 (2017).

44. Amstutz, U., Froehlich, T. K. \& Largiadèr, C. R. Dihydropyrimidine dehydrogenase gene as a major predictor of severe 5-fluorouracil toxicity. Pharmacogenomics. 12, 1321-36 (2011).

45. Lunenburg, C., Swen, J., Guchelaar, H. J. \& Gelderblom, H. Capecitabine-induced severe toxicity secondary to DPD deficiency and successful treatment with low dose 5-Fluorouracil. J Gastrointest Cancer. 48, 117-8 (2017).

46. Caudle, K. E. et al. Clinical Pharmacogenetics Implementation Consortium guidelines for dihydropyrimidine dehydrogenase genotype and fluoropyrimidine dosing. Clin Pharmacol Ther. 94, 640-5 (2013).

47. XELODA ${ }^{\circledR}$ (Capecitabine) [package insert on the internet]. South San Francisco: Hoffmann-La Roche, Inc.; [revised 2015 Mar]. Available from: https://www.accessdata.fda.gov/drugsatfda_docs/label/2015/020896s036lbl.pdf (1998).

48. Fluorouracil (injection) [package insert on the internet]. Irvine: Spectrum Pharmaceuticals, Inc.; [revised 2016 Jul]. Available from: https://www.accessdata.fda.gov/drugsatfda_docs/label/2016/012209s040lbl.pdf (1962).

49. Carac $^{\circledR}$ (Fluorouracil cream) [package insert on the internet]. Berwyn: Dermik Laboratories, Inc.; Available from: https://www. accessdata.fda.gov/drugsatfda_docs/label/2003/20985slr004_carac_lbl.pdf (2003).

\section{Acknowledgements}

We thank Helber Palheta for technical assistance. We acknowledge funding from CNPq (Conselho Nacional de Desenvolvimento Científico e Tecnológico), UFPA (Universidade Federal do Pará) and CAPES (Coordenação de Aperfeiçoamento de Pessoal de Nível Superior). The funders had no role in study design, data collection and analysis, decision to publish, or preparation of the manuscript.

\section{Author Contributions}

J.C.G.R., M.R.F. and N.P.C.S. designated the study. J.C.G.R. and M.R.F. conducted the molecular genetic study, participated in the statistical analyses, and wrote the manuscript. J.F.G. collected the Amerindians' blood samples and participated in the design of the study. A.L.C.S. assisted in the research methodology. Â.R.S., S.S. and N.P.C.S. were the coordinators of the project. All the authors have read and approved the final manuscript.

\section{Additional Information}

Supplementary information accompanies this paper at https://doi.org/10.1038/s41598-019-43610-y.

Competing Interests: The authors declare no competing interests.

Publisher's note: Springer Nature remains neutral with regard to jurisdictional claims in published maps and institutional affiliations.

Open Access This article is licensed under a Creative Commons Attribution 4.0 International License, which permits use, sharing, adaptation, distribution and reproduction in any medium or format, as long as you give appropriate credit to the original author(s) and the source, provide a link to the Creative Commons license, and indicate if changes were made. The images or other third party material in this article are included in the article's Creative Commons license, unless indicated otherwise in a credit line to the material. If material is not included in the article's Creative Commons license and your intended use is not permitted by statutory regulation or exceeds the permitted use, you will need to obtain permission directly from the copyright holder. To view a copy of this license, visit http://creativecommons.org/licenses/by/4.0/.

(C) The Author(s) 2019 